\title{
Efficient virus-induced gene silencing in Hibiscus hamabo Sieb. et Zucc. using tobacco rattle virus
}

\author{
Zhiquan Wang ${ }^{1}$, Xiaoyang Xu ${ }^{1}$, Longjie $\mathrm{Ni}^{1,2}$, Jinbo Guo ${ }^{1}$, Chunsun Gu ${ }^{\text {Corresp. } 1}$ \\ 1 Jiangsu Key Laboratory for the Research and Utilization of Plant Resources, Institute of Botany, Jiangsu Province and Chinese Academy of Sciences, \\ Nanjing, Jiangsu, China \\ ${ }^{2}$ College of Forest Sciences, Nanjing Forestry University, Nanjing, Jiangsu, China \\ Corresponding Author: Chunsun Gu \\ Email address: chunsungu@cnbg.net
}

Background. Hibiscus hamabo Sieb.et Zucc. is a semi-mangrove plant used for the ecological restoration of saline-alkali land, coastal afforestation and urban landscaping. The genetic transformation $\mathrm{H}$. hamabo is currently inefficient and laborious, restricting gene functional studies on this species. In plants, virus-induced gene silencing provides a pathway to rapidly and effectively create targeted gene knockouts for gene functional studies. Methods. In this study, we tested the efficiency of a tobacco rattle virus vector in silencing the cloroplastos alterados 1 (CLA1) gene through agroinfiltration. Results. The leaves of $H$. hamabo showed white streaks typical of CLAl gene silencing three weeks after agroinfiltration. In agroinfiltrated $H$. hamabo plants, the CLAl expression levels in leaves with white streaks were all significantly lower than those in leaves from mockinfected and control plants. Conclusions. The system presented here can efficiently silence genes in $\mathrm{H}$. hamabo and may be a powerful tool for large-scale reverse-genetic analyses of gene functions in $H$. hamabo. 
1 Efficient virus-induced gene silencing in Hibiscus

2 hamabo Sieb. et Zucc. using tobacco rattle virus

3

4

5

6

7

8

9

10

11

12

13

14

15

16

17

18

19

20

21

22

23

24

25

26

27

28

29

\author{
Zhiquan Wang ${ }^{1}$, Xiaoyang $\mathrm{Xu}^{1}$, Longjie $\mathrm{Ni}^{1,2}$, Jinbo Guo ${ }^{1}$, Chunsun $\mathrm{Gu}^{1 *}$
}

${ }^{1}$ Jiangsu Key Laboratory for the Research and Utilization of Plant Resources, Institute of Botany, Jiangsu Province and Chinese Academy of Sciences, Nanjing, Jiangsu, China.

${ }^{2}$ College of Forest Sciences, Nanjing Forestry University, Nanjing, Jiangsu, China.

Corresponding Author:

Chunsun $\mathrm{Gu}^{1}$

1 Qianhuhoucun Road, Nanjing, Jiangsu, 210014, China

Email address: chunsungu@cnbg.net

\title{
Abstract
}

Background. Hibiscus hamabo Sieb.et Zucc. is a semi-mangrove plant used for the ecological restoration of saline-alkali land, coastal afforestation and urban landscaping. The genetic transformation $H$. hamabo is currently inefficient and laborious, restricting gene functional studies on this species. In plants, virus-induced gene silencing provides a pathway to rapidly and effectively create targeted gene knockouts for gene functional studies.

Methods. In this study, we tested the efficiency of a tobacco rattle virus vector in silencing the cloroplastos alterados 1 (CLAl) gene through agroinfiltration.

Results. The leaves of $H$. hamabo showed white streaks typical of $C L A 1$ gene silencing three weeks after agroinfiltration. In agroinfiltrated $H$. hamabo plants, the CLAl expression levels in leaves with white streaks were all significantly lower than those in leaves from mock-infected and control plants.

Conclusions. The system presented here can efficiently silence genes in H. hamabo and may be a powerful tool for large-scale reverse-genetic analyses of gene functions in H. hamabo.

\section{Introduction}

Hibiscus hamabo Sieb.et Zucc., which is a shrub plant in the genus Hibiscus, family Malvaceae, is an important semi-mangrove plant (Nakanishi 1979). Because of its excellent salt tolerance and morphological characteristics, H. hamabo is widely used in public parks, waysides and coastal sands near sea level (Fowler 2017; Li et al. 2012; Yang et al. 2008). In addition, $H$. hamabo is a good plant material for exploring the salt-stress response mechanisms of woody plants (Li et al. 2012). Gene manipulation technologies can be used to determine the gene functions and regulatory mechanisms in H. hamabo. However, to date, the inefficient and laborious genetic transformation procedures used have impeded such research. Additionally, 
39

40

41

42

43

44

45

46

47

48

49

50

51

52

53

54

55

56

57

58

59

60

61

62

63

64

65

66

67

68

69

70

71

72

73

74

75

76

77

78

transcriptome analyses have mined many excellent genes that are awaiting functional identification. Appropriate techniques need to be applied successfully to allow the study of gene functions in this plant.

Virus-induced gene silencing (VIGS) is a powerful technology that uses engineered viruses to specifically silence host gene expression through post-transcriptional gene silencing (Becker \& Lange 2010; Krishnan et al. 2015; Purkayastha \& Dasgupta 2009). VIGS is an effective method for large-scale analysis of genes and their functions, and it has been successfully performed in many plants, including tobacco, Arabidopsis, tomato, cotton, wheat, and many woody plants (Burch-Smith et al. 2006; Jiang et al. 2014; Kumagai et al. 1995; Orzaez et al. 2009; Scofield et al. 2005). VIGS works via a mechanism that is similar to that of RNA interference (Baulcombe 1999; Baulcombe 2004; Burch-Smith et al. 2004; Lu et al. 2003; Waterhouse et al. 2001). Double-stranded (ds) RNA is the key to the VIGS process; the dsRNA can be cleaved into short interfering (si) RNAs of 21 to 25 nucleotides (Burch-Smith et al. 2004; Jiang et al. 2014; Lu et al. 2003). Two strands can be obtained from the siRNAs - the guide and passenger strands. The RNA-induced silencing complex incorporates the guide strand to degrade the specific single-stranded RNA that is complementary to the guide RNA, and then, the passenger strand is degraded (Mustafa et al. 2016). As a result, the target gene is silenced and large amounts of siRNAs are produced (Fuchs et al. 2004).

Agrobacterium-mediated VIGS protocols based on tobacco rattle virus (TRV) have been developed and optimized in cotton, and previous studies showed that TRV is a useful vector for VIGS in Gossypium species (Gao et al. 2011; Ge et al. 2016). Tobacco rattle virus (TRV), belonging to genus Tobravirus (family Virgaviridae), is a suitable virus vector system for VIGS (Jiang et al. 2014). A positive sense single-stranded RNA genome exists in TRV, consisting of two components, RNA 1 and RNA 2 (Mustafa et al. 2016). RNA 1 encodes genes with viral replication and movement functions, while RNA 2 encodes the coat protein and some nonessential structural proteins that can be replaced by foreign sequences (Hayward et al. 2011). The TRV vector has been used in G. spp., Arabidopsis and Vernicia fordii to silence the cloroplastos alterados $1(C L A 1)$ gene, which is involved in chloroplast development (Jiang et al. 2014; Manhães et al. 2015; Mustafa et al. 2016). The CLA1 gene is highly conserved in various plant species (Jiang et al. 2014). The silencing phenotypes of albino leaves were observed in Vernicia fordii two weeks after inoculation using a heterologous TRV-based VIGS system, in which CLA1 was isolated from Populus tomentosa Carr. (Jiang et al. 2014). The silenced CLA1 is a useful marker for determining silencing efficiency because of the bleached phenotype (Mustafa et al. 2016).

In this study, we tested the feasibility of the TRV-VIGS system in H. hamabo using the HhCLAl gene as a reporter. The agroinfiltrated leaves of $H$. hamabo showed white streaks typical at three weeks after infection, and the expression levels of the HhCLAl gene in leaves with white streaks were significantly lower than those in leaves from mock-infected and control plants. Thus, the TRV-VIGS system can efficiently silence genes in H. hamabo. To our knowledge, this is the first report of the successful application of VIGS in H. hamabo. 
79

80

81

82

83

84

85

86

87

88

89

90

91

92

93

94

95

96

97

98

99

100

101

102

103

104

105

106

107

108

109

110

111

112

113

114

115

116

117

118

\section{Materials \& Methods}

\section{Plant materials and growth conditions.}

Seeds of H. hamabo were collected from Nanjing's Sun Yat-Sen Memorial Botanical Garden. The seeds were then treated with concentrated sulfuric acid for $15 \mathrm{~min}$ and rinsed thoroughly with sterile water. The pretreated seeds were sown into flowerpots containing a mixture of peat and vermiculite $(1: 1, \mathrm{v}: \mathrm{v})$ in an illuminated incubator with controlled temperatures of $26^{\circ} \mathrm{C} / 22$ ${ }^{\circ} \mathrm{C}$ under a $16 \mathrm{~h} / 8 \mathrm{~h}$ (day/night) photoperiod.

\section{Sequence analysis.}

Based on the HhCLA1 sequence (GenBank accession no. MK229167), the deduced protein sequence was analyzed with CLA1 proteins of other species using ClustalX (Liu et al. 2015). The amino acid sequences were obtained from NCBI (https://www.ncbi.nlm.nih.gov/). Then, the sequences were used to construct a phylogenetic tree, which was drawn with MEGA 7.0 using the Neighbor-Joining (NJ) method and 1,000 bootstrap replicates.

\section{VIGS vector construction.}

Total RNA was extracted from the leaves of $H$. hamabo using a Plant RNeasy Mini Kit (Qiagen, Hilden, Germany). The first-strand cDNA was synthesized using a SuperScript II reverse transcriptase kit (TaKaRa, Dalian, China). The primer pair HhCLA1-F and HhCLA1-R (Table 1) was designed using Oligo 6.0 software (Molecular Biology Insights, Inc., Cascade, CO, USA) based on the conserved domain of HhCLA1. To amplify partial fragments of HhCLA1, the primer pair, cDNA and PrimeSTAR ${ }^{\mathrm{TM}}$ HS DNA polymerase (TaKaRa) were used. EcoRI enzyme cleavage sites were added to the upstream primers and SacI enzyme cleavage sites were added to the downstream primers. PCR product were generated with the following reaction program: 30 cycles of $98^{\circ} \mathrm{C}$ for $10 \mathrm{~s}, 60^{\circ} \mathrm{C}$ for $5 \mathrm{~s}$ and $72^{\circ} \mathrm{C}$ for $1 \mathrm{~min}$. The reactions final volume was $50 \mu \mathrm{L}$, containing $25 \mu \mathrm{L}$ of $2 \times$ PrimeSTAR ${ }^{\mathrm{TM}}$ GC Buffer, $4 \mu \mathrm{L}$ dNTP mixture $(2.5 \mathrm{mM}), 0.2 \mu \mathrm{M}$ of each primer (final), $100 \mathrm{ng}$ of cDNA and $0.5 \mu \mathrm{L}$ of PrimeSTAR ${ }^{\mathrm{TM}}$ HS DNA Polymerase (2.5 $\mathrm{U} / \mu \mathrm{L}$ ). The $p T R V 1$ and $p T R V 2$ vectors were used in this study as described previously (Gao et al. 2011; Liu et al. 2002). The PCR products were ligated into pTRV2 (Supplementary Fig. S1) (double-digested with $E c o R I$ and $S a c I$ enzymes) using a ClonExpress ${ }^{\circledR}$ IIOne Step Cloning Kit (Vazyme, Nanjing, China). The resulting vector was designated pTRV2-HhCLA1.

\section{Agroinfiltration.}

pTRV2-HhCLA1 was transformed into Agrobacterium tumefaciens strain 'GV3101' using the freeze-thawing method (Höfgen \& Willmitzer 1988). PCR-confirmed single colonies were then selected and independently inoculated into $3 \mathrm{~mL}$ of Luria-Bertani medium containing $25 \mathrm{mg} / \mathrm{L}$ rifampicin and $50 \mathrm{mg} / \mathrm{L}$ kanamycin and grown overnight in a shaker at $28^{\circ} \mathrm{C}$. For the VIGS assay, 3-mL cultures of A. tumefaciens strain GV3101 independently containing either pTRV1 or pTRV2 was grown overnight in the same culture conditions. These overnight starter cultures were subsequently used to inoculate $50-\mathrm{mL}$ cultures that were grown overnight at $28^{\circ} \mathrm{C}$. Agrobacterium cultures were harvested by centrifugation at $4,000 \times \mathrm{g}$ for $10 \mathrm{~min}$, and the pellets were resuspended in an infiltration buffer (10 mM MES (2- (4- Morpholino) Ethanesulfonic 
119 Acid), $10 \mathrm{mM} \mathrm{MgCl} 2$ and $200 \mu \mathrm{M}$ acetosyringone, $\mathrm{pH} 5.6)$ at an optical density of 2.0 at $600 \mathrm{~nm}$ 120 and incubated at room temperature for $3 \mathrm{~h}$ without shaking. Agrobacterium cultures containing 121 mixtures of pTRV1 and pTRV2-HhCLA1 (1: 1 ratio) were infiltrated with 1-mL needleless 122 syringes into the backs of cotyledons of 2-week-old $H$. hamabo seedlings, following a protocol 123 described previously (Gao et al. 2011). To determine whether the TRV vector can directly infect H. hamabo, a mixture of Agrobacterium cultures containing pTRV1 and pTRV2 constructs in a 1: 1 ratio was infiltrated into the backs of cotyledons of eight 2-week-old $H$. hamabo to serve as the mock. Experimental and non-injected control plants were transferred to a growth chamber and maintained under set conditions.

To determine the relative levels of the endogenous HhCLA1 transcripts in infected leaves exhibiting visible silencing phenotypes, qPCR was performed using the primer pair qHhCLA1-

131

132

133

134

135

136

137

138

139

140

141

142

143

144

145

146

147

148

149

150

151

152

153

154

155

156

157

158

F/qHhCLA1-R (Table 1). For the experiments, leaves from plants with significant white streak symptoms were analyzed in comparison with leaves of the mock and control plants after three weeks of agroinfiltration. Four groups of plants with significant white streak symptoms, one control group, and one mock group, in order to analyze the test results more accurately, were further analyzed in this experiment. Each group contained three biological replicates. Total RNA was extracted from these leaves using a Plant RNeasy Mini Kit (Qiagen) and treated with DNase I to remove residual DNA. The first-strand cDNA was synthesized using a SuperScript II reverse transcriptase kit (TaKaRa). The qPCR assays were performed using the SYBR Green PCR Master Mix (Bimake, Houston, TX, USA) and a StepOne ${ }^{\text {TM }}$ System (ABI, USA). The transcript level of $18 S \mathrm{rRNA}$ served as the internal controls. All experiments were repeated three times. The relative gene expression level was calculated using the $2^{-\Delta \Delta \mathrm{Ct}}$ method (Gu et al. 2018; Liao et al. 2016).

\section{Statistical analysis.}

One-way analysis of variance (ANOVA) and Duncan's multiple range test $(P<0.05)$ were performed using IBM SPSS (Version 21).

\section{Results}

\section{Characterization of the $H h C L A 1$ gene in $H$. hamabo.}

The amino acid sequence alignment indicated that the HhCLA1 protein showed high homology to known CLA1 proteins from G. barbadense, G. hirsutum and other species (Fig. 1 and Supplementary Table S1). The phylogenetic analysis showed that HhCLA1 clustered with $G$. barbadense and G. hirsutum in a clade (Fig. 2).

\section{Silencing efficiency of the $H h C L A 1$ gene in $H$. hamabo using the VIGS system.}

In total, fifty-two $H$. hamabo plants were inoculated with A. tumefaciens 'GV3101' harboring pTRV2-HhCLA1. Two weeks after agroinfiltration, white streaks began to appear in the emerging leaves of partially agroinoculated plants. At three weeks post infiltration, $87 \%$ of the H. hamabo plants showed white-streak leaf symptoms similar to the photobleached phenotype (Table 2; Fig. 3A and Supplementary Fig. S2). At three weeks after agroinfiltration, plants 
159

160

161

162

163

164

165

166

167

168

169

170

171

172

173

174

175

176

177

178

179

180

181

182

183

184

185

186

187

188

189

190

191

192

193

194

195

196

197

inoculated with pTRV1 and pTRV2 (Mock) showed no obvious differences in leaf morphology compared with the control (Fig. 3B, 3C and Supplementary Fig. S2). The leaves in Fig. 3D are from plants infiltrated with pTRV2-HhCLA1 (CLA1), empty vector infiltrated plant (Mock) and the control plant (CK) separately. Leaf phenotypic characteristics suggested that the HhCLA1 gene expression might be suppressed in plants infiltrated with pTRV2-HhCLA1 compared with mock and CK plants.

\section{q-PCR analysis of the knockdown levels of $H \boldsymbol{h C L A 1 .}$}

The efficiency of gene silencing was analyzed by monitoring expression levels of HhCLA1 in plants showing white-streak leaf symptoms. Results showed that HhCLAl gene expression levels were unchanged in mock-injected plants, while the HhCLA1 expression levels were $62.6 \%$ $76.4 \%$ lower in the pTRV2-HhCLA1 agroinfiltrated plants than in the non-infiltrated plants (control) (Fig. 4 and Table S2). The phenotypic characteristics were consistent with the expression characteristics of HhCLA1. This clearly indicates that the expression of HhCLAl was significantly down-regulated through TRV-VIGS in H. hamabo, and TRV-VIGS led to an albino phenotype on leaves.

\section{Discussion}

In this study, we demonstrated for the first time that TRV-VIGS can effectively down-regulate endogenous gene expression levels in the salt-tolerant species $H$. hamabo. The genetic transformation of this species is currently laborious, time-consuming and technically challenging. To resolve these problems, effective and low-cost techniques need to be developed to enable the rapid validation of gene functions. In future studies, stress-responsive genes isolated in H. hamabo could be silenced in loss-of-function screens using the TRV-VIGS system.

The CLA1 gene is involved in chloroplast development and is a useful marker in the TRVVIGS system (Mustafa et al. 2016). In this research, multiple sequence alignments indicated that HhCLA1 was similar to CLA1 proteins of other species. Additionally, the phylogenetic analysis indicated that HhCLA1 was highly similar to CLA1 proteins in Malvaceae, including $G$. barbadense and G. hirsutum.

The most cost-efficient and effective method of inoculating plants with virus-based vectors is agroinfection (Grimsley et al. 1986), but its efficiency varies among plants (Zhang et al. 2016). In turf grass, the silencing efficiency of the RTBV-VIGS system in Cynodon dactylon was such that $65.8 \%$ - $72.5 \%$ of the agroinfected plants developed symptoms typical for phytoene desaturase gene silencing, while the silencing efficiency in Zoysia japonica was much lower, with only $52.7 \%-55 \%$ of agroinfected plants developing the phenotype (Zhang et al. 2016). The ability of the TRV vector to directly infect woody plant species has been tested, and TRVmediated VIGS was effective in Vernicia fordii, weak in Populus tomentosa Carr., and ineffective in Camellia oleifera (Jiang et al. 2014). In this study, the silencing efficiency of the TRV-VIGS system in $H$. hamabo was high, with $87 \%$ of agroinfected plants developing a white- 
198

199

200

201

202

203

204

205

206

207

208

209

210

211

212

213

214

215

216

217

218

219

220

221

222

223

224

225

226

227

228

229

230

231

232

233

234

235

236

237

238

239

240

241

streak leaf phenotype. The HhCLA1 mRNA level was also down-regulated by TRV-VIGS in $H$. hamabo.

\section{Conclusions}

In conclusion, we demonstrated that TRV-mediated VIGS can effectively silence genes in $H$. hamabo, which adds to the increasing list of wood species for which VIGS-mediated studies can be used. The loss-of-function assay using TRV-mediated VIGS developed in this study provides an alternative tool for functional genes studies of $H$. hamabo.

\section{Acknowledgements}

We thank International Science Editing for editing this manuscript.

\section{References}

Baulcombe D. 1999. Viruses and gene silencing in plants. 100 Years of Virology: Springer, 189201.

Baulcombe D. 2004. RNA silencing in plants. Nature 431(7006): 356.

Becker A, and Lange M. 2010. VIGS-genomics goes functional. Trends in Plant Science 15: 1-4. Burch-Smith TM, Schiff M, Liu Y, and Dinesh-Kumar SP. 2006. Efficient virus-induced gene silencing in Arabidopsis. Plant Physiology 142: 21-27.

Burch-Smith TM, Anderson JC, Martin GB, and Dinesh-Kumar S. P. 2004. Applications and advantages of virus - induced gene silencing for gene function studies in plants. Plant Journal 39(5): 734-746.

Fowler E. 2017. Assessment and characterization of microbial communities in salt affected soils on Galveston Island. SFA scholarworks, Electronic Theses and Dissertations. 76, Stephen F Austin State University.

Fuchs U, Damm-Welk C, and Borkhardt A. 2004. Silencing of disease-related genes by small interfering RNAs. Current Molecular Medicine 4: 507-517.

Gao X, Britt Jr RC, Shan L, and He P. 2011. Agrobacterium-mediated virus-induced gene silencing assay in cotton. Journal of Visualized Experiments: JoVE 54: e2938.

Ge X, Wu J, Zhang C, Wang Q, Hou Y, Yang Z, Yang Z, Xu Z, Wang Y, Lu L, Zhang X, Hua J, and Li F. 2016. Prediction of VIGS efficiency by the Sfold program and its reliability analysis in Gossypium hirsutum. Science Bulletin 61(7): 543-551.

Grimsley N, Hohn B, Hohn T, and Walden R. 1986. "Agroinfection," an alternative route for viral infection of plants by using the Ti plasmid. Proceedings of the National Academy of Sciences 83:3282-3286.

Gu C, Liu L, Song A, Liu Z, Zhang Y, and Huang S. 2018. Iris lactea var. chinensis (Fisch.) cysteine-rich gene $l l C D T 1$ enhances cadmium tolerance in yeast cells and Arabidopsis thaliana. Ecotoxicology and Environmental Safety 157:67-72.

Höfgen R, and Willmitzer L. 1988. Storage of competent cells for Agrobacterium transformation. Nucleic Acids Research 16:9877.

Hayward A, Padmanabhan M, and Dinesh-Kumar S. 2011. Virus-induced gene silencing in Nicotiana benthamiana and other plant species. Plant Reverse Genetics: Springer 55-63.

Jiang Y, Ye S, Wang L, Duan Y, Lu W, Liu H, Fan D, Zhang F, and Luo K. 2014. Heterologous gene silencing induced by tobacco rattle virus (TRV) is efficient for pursuing functional

PeerJ reviewing PDF | (2019:02:34799:4:0:ACCEPTED 4 Jul 2019) 
242

243

244

245

246

247

248

249

250

251

252

253

254

255

256

257

258

259

260

261

262

263

264

265

266

267

268

269

270

271

272

273

274

275

276

277

278

279

280

281

282

283

284

genomics studies in woody plants. Plant Cell Tissue \& Organ Culture 116:163-174.

Krishnan A, Mahadevan C, Mani T, and Sakuntala M. 2015. Virus-induced gene silencing (VIGS) for elucidation of pathogen defense role of serine/threonine protein kinase in the non-model plant Piper colubrinum Link. Plant Cell Tissue \& Organ Culture 122:269-283.

Kumagai M, Donson J, Della-Cioppa G, Harvey D, Hanley K, and Grill L. 1995. Cytoplasmic inhibition of carotenoid biosynthesis with virus-derived RNA. Proceedings of the National Academy of Sciences 92:1679-1683.

Li J, Liao J, Guan M, Wang E, and Zhang J. 2012. Salt tolerance of Hibiscus hamabo seedlings: a candidate halophyte for reclamation areas. Acta Physiologiae Plantarum 34:1747-1755.

Liao YL, Xu F, Zang WW, Cheng SY, Shen YB, and Chang J. 2016. Cloning, characterization and expression analysis of GbWRKY11, a novel transcription factor gene in Ginkgo biloba. International Journal of Agriculture and Biology 18(1): 117-124.

Liu C, Wang G, Wang H, Xia T, Zhang S, Wang Q, and Fang Y. 2015. Phylogenetic relationships in the genus Rosa revisited based on rpl16, trnL-F, and atpB-rbcL sequences. HortScience 50(11): 1618-1624.

Liu Y, Schiff M, and Dinesh-Kumar S. 2002. Virus-induced gene silencing in tomato. The Plant Journal 31: 777-786.

Lu R, Martin-Hernandez AM, Peart J R, Malcuit I, and Baulcombe DC. 2003. Virus-induced gene silencing in plants. Methods 30(4): 296-303.

Manhães AMEdA, de Oliveira MV, and Shan L. 2015. Establishment of an Efficient VirusInduced Gene Silencing (VIGS) Assay in Arabidopsis by Agrobacterium-Mediated Rubbing Infection. Plant Gene Silencing: Springer 235-241.

Mustafa R, Shafiq M, Mansoor S, Briddon RW, Scheffler BE, Scheffler J, and Amin I. 2016. Virus-induced gene silencing in cultivated cotton (Gossypium spp.) using tobacco rattle virus. Molecular Biotechnology 58:65-72.

Nakanishi H. 1979. Distribution and ecology of a semi-mangrove plant, Hibiscus hamabo SIEB. et ZUCC. and its community. Acta Phytotaxonomica Et Geobotanica 30:169-179.

Orzaez D, Medina A, Torre S, Fernández-Moreno JP, Rambla JL, Fernández-del-Carmen A, Butelli E, Martin C, and Granell A. 2009. A visual reporter system for virus-induced gene silencing in tomato fruit based on anthocyanin accumulation. Plant Physiology 150:11221134.

Purkayastha A, and Dasgupta I. 2009. Virus-induced gene silencing: a versatile tool for discovery of gene functions in plants. Plant Physiology and Biochemistry 47:967-976.

Scofield SR, Huang L, Brandt AS, and Gill BS. 2005. Development of a virus-induced genesilencing system for hexaploid wheat and its use in functional analysis of the Lr21mediated leaf rust resistance pathway. Plant Physiology 138:2165-2173.

Waterhouse PM, Wang MB, and Lough T. 2001. Gene silencing as an adaptive defence against viruses. Nature 411:834.

Yang H, Du GJ, and Wang KH. 2008. Study on the Physiological Characteristics of Hibiscus hamabo under Stress. Journal of Zhejiang Forestry Science and Technology 3:011.

Zhang B, Shi J, Chen J, Li D, Li J, Guo H, Zong J, Wang Y, Guo A, and Liu J. 2016. Efficient virus-induced gene silencing in Cynodon dactylon and Zoysia japonica using rice tungro bacilliform virus vectors. Scientia Horticulturae 207:97-103.

Peer] reviewing PDF | (2019:02:34799:4:0:ACCEPTED 4 Jul 2019) 


\section{Figure 1}

Multiple alignment of the HhCLAl amino acid sequence with sequences from different species using the ClustalW program.

Multiple alignment of protein sequences of the HhCLA1 gene in Hibiscus hamabo Sieb. et Zucc., Gossypium barbadense (ABN13970.1), Gossypium hirsutum (NP_001314056.1), Theobroma cacao (EOY06359.1), Arabidopsis thaliana (NP_193291.1) and Populus tomentosa (AGT02336.1).
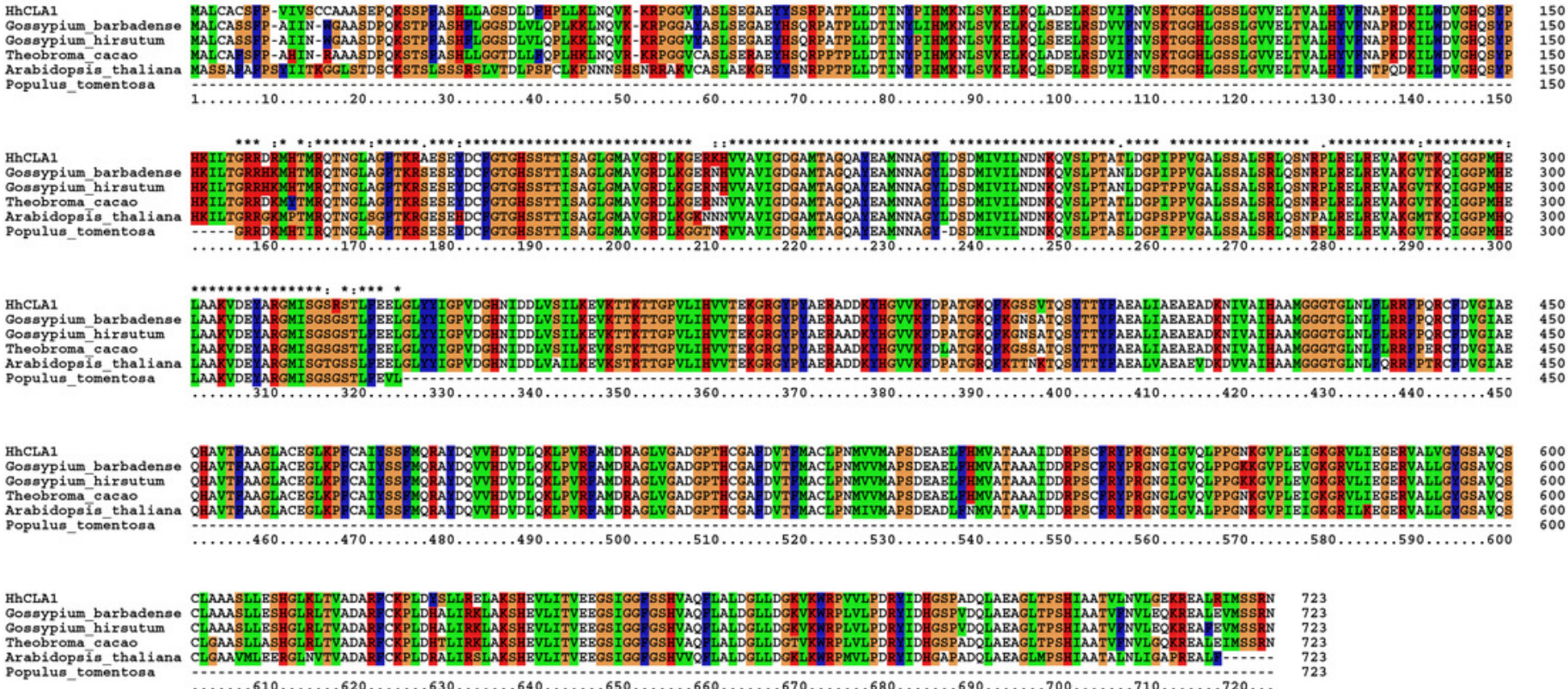
Figure 2 (on next page)

Phylogenetic analysis of the protein of HhCLA1.

Phylogenetic analysis of HhCLA1 proteins in different species. 


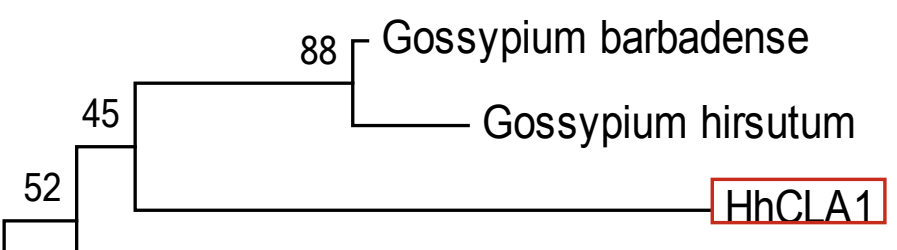

Populus tomentosa

Theobroma cacao

Arabidopsis thaliana

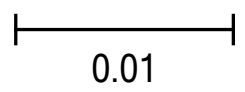




\section{Figure 3}

TRV-induced HhCLAl silencing in H. hamabo.

(A) Newly formed leaves of $H$. hamabo plants infiltrated with pTRV2-HhCLA1 (CLA1) showing white-streaked leaf symptoms after three weeks. (B) Empty vector infiltrated plants (Mock) with the normal phenotype. (C) Control plants (CK). (D) The leaf phenotypes of the treatments. The three leaves on the left in Fig. 3D are from plants infiltrated with pTRV2HhCLA1 (CLA1), the leaf in the middle is from an empty vector infiltrated plant (Mock) and the right one is from a control plant (CK). 


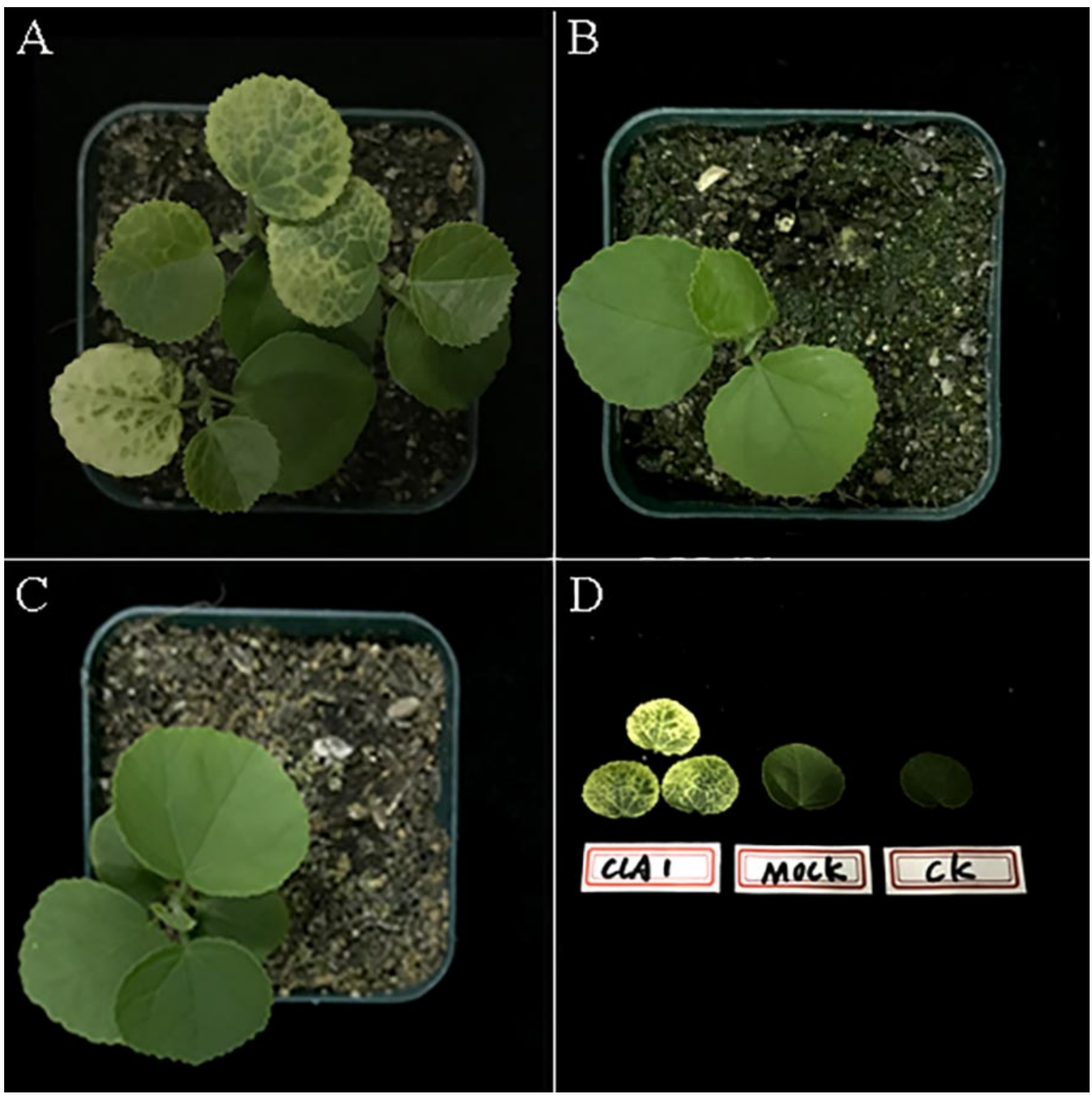


Figure 4 (on next page)

Relative expression levels of HhCLA1 transcripts in control (CK), empty vector-infected (Mock) and pTRV-HhCLA1-infected plants (pTRV2-HhCLA1).

Error bars represent standard errors, and any two samples with a common capital letter are not significantly different at the $P<0.01$ level, as with a same small letter are not significantly different at the $P<0.05$ level. 


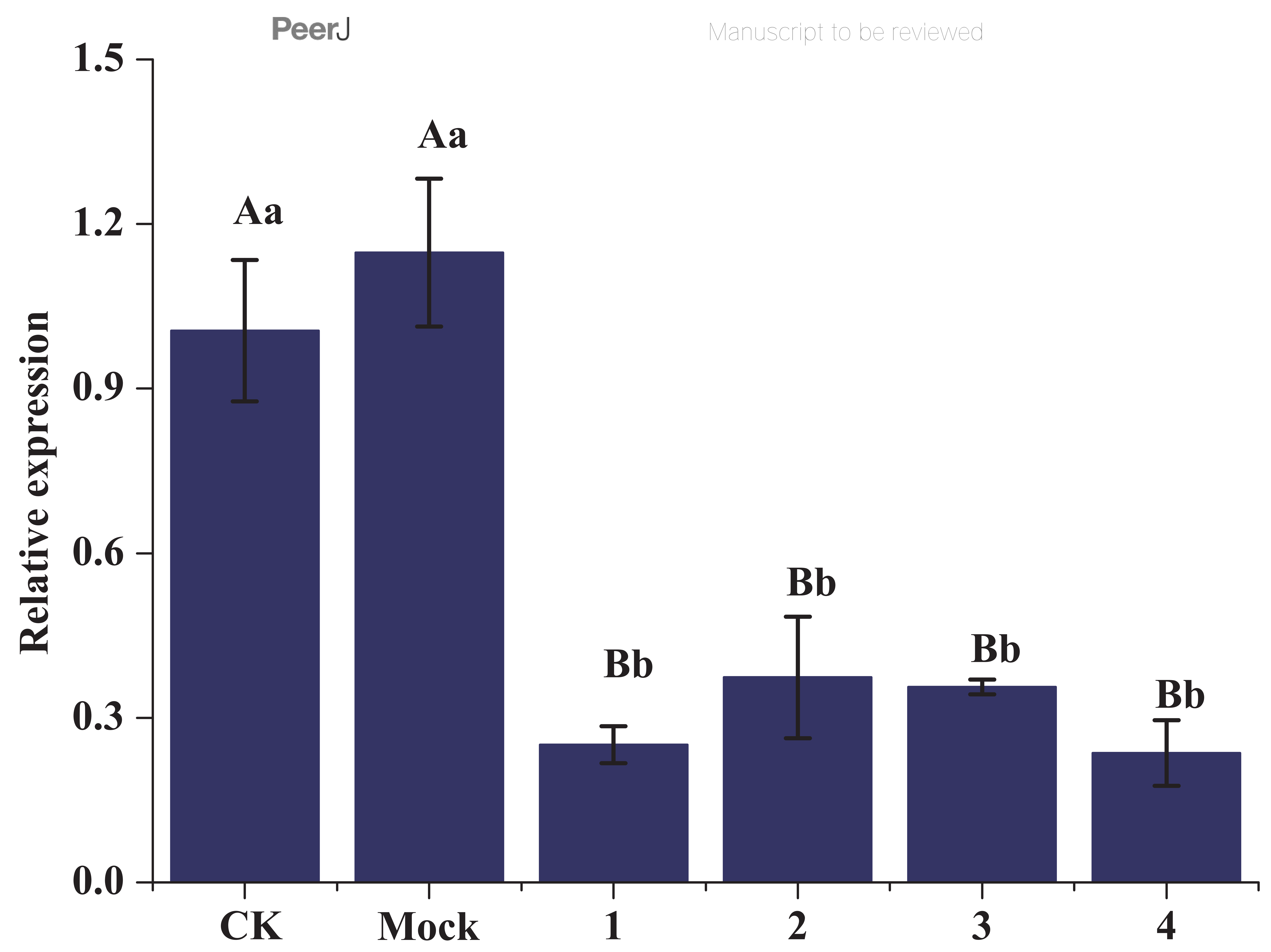




\section{Table 1 (on next page)}

Primers used in this TRV-VIGS system.

Note: Underlines indicate restriction enzyme cleavage sites used in this TRV-VIGS system. 
1 Table 1 Primers used in this TRV-VIGS system

\begin{tabular}{|c|c|}
\hline Primer Name & Primer Sequence \\
\hline HhCLA1-F & CTGTGAGTAAGGTTACCGAATTCTCATGTTGTCACTGAGAAAGG \\
\hline HhCLA1-R & $\underline{\text { CTCGAGACGCGTGAGCTCCATAGCAAATCTTACAGGCAG }}$ \\
\hline$q H h C L A 1-\mathrm{F}$ & CGCCAGGGAACAAAGGGGTT \\
\hline$q H h C L A 1-\mathrm{R}$ & AATCGTGCATCCGCGACAGT \\
\hline $18 S \mathrm{rRNA}-\mathrm{F}$ & GGTCGGATTTGGAACGGCGA \\
\hline $18 S \operatorname{rRNA} A \mathrm{R}$ & CTCCACGGGCGTATCGAGG \\
\hline
\end{tabular}

2 Note: Underlines indicate restriction enzyme cleavage sites used in this TRV-VIGS system. 


\section{Table 2 (on next page)}

Efficiency of HhCLA1 gene silencing in Hibiscus hamabo using TRV-VIGS system at three weeks post agroinfiltration.

"Silencing efficiency indicates the number of plants showing silencing phenotypes/number of plants treated by TRV-VIGS system. 
1 Table 2 Efficiency of HhCLA1 gene silencing in Hibiscus hamabo using TRV-VIGS system at

2 three weeks post agroinfiltration

\begin{tabular}{lll}
\hline Treatment & Number of plants assayed & Silencing efficiency $^{*}$ \\
\hline pTRV2-HhCLA1 & 52 & $45 / 52(87 \%)$ \\
Mock & 8 & $0 / 8(0 \%)$ \\
Control & 8 & $0 / 8(0 \%)$ \\
\hline
\end{tabular}

3 * Silencing efficiency indicates the number of plants showing silencing phenotypes/number of

4 plants treated by TRV-VIGS system.

5 